\title{
A Study on Clinical Profile and Common Comorbid Conditions among Chronic Obstructive Pulmonary Disease (COPD) Patients in a Tertiary Care Hospital in Eastern India
}

\author{
Sanjay Dhali ${ }^{1}$, Tapan Das Bairagya ${ }^{2}$ \\ ${ }^{1}$ Department of Chest Medicine, North Bengal Medical College and Hospital, Darjeeling, West Bengal, India. \\ ${ }^{2}$ Department of Chest Medicine, North Bengal Medical College and Hospital, Darjeeling, West Bengal, India.
}

\section{ABSTRACT}

\section{BACKGROUND}

Chronic Obstructive Pulmonary Disease (COPD) is a disorder that is characterized by progressive airflow limitation that is not fully reversible, with important extrapulmonary manifestation. In practice, this is measured as the $\mathrm{FEV}_{1} / \mathrm{FVC}$ ratio and the arbitrary definition of airflow obstruction is generally taken to be an FEV $1 / F V C$ ratio lower than 0.70 . The objective of the current study is to find out clinical presentation and asses the comorbid condition along with chronic obstructive pulmonary disease (COPD) patients.

\section{METHODS}

A one-year observational study was planned to assess the diagnosis and clinical presentation of COPD patients along with comorbidity as per GOLD-2009 guidelines.

\section{RESULTS}

One hundred patients of Chronic Obstructive Pulmonary Disease (COPD) were included in our study. Among them, 84 were males (84\%) and 16 were females $(16 \%)$. Most of the patients were in above-50-years age group (as $89 \%$ male vs. $87.5 \%$ female) and among these, most of the patients were above 65 years ( $48 \%$ male vs. $50 \%$ female). The most common presenting complaint was shortness of breath. $10.7 \%$ of male and $18.75 \%$ of female COPD patients were found to have anaemia. Anaemia of chronic disease and iron deficiency anaemia was commonly occurrence. In our study, it was found that, 14 cases were having diabetes (14\%) and 20 cases were having hypertension (20\%). None of the patients in my study suffered from both diabetes and hypertension. In this study, 6 (7\%) male COPD patients had ischemic heart disease and $12.5 \%$ of female patients had ischemic heart disease.

\section{CONCLUSIONS}

Shortness of breath was the most common presentation. Males are more prone to develop COPD. Diabetes, hypertension, anaemia, ischemic heart disease were found to be the co-morbid conditions.

\section{KEY WORDS}

Chronic Obstructive Pulmonary Disease, Diabetes, Comorbidity, Spirometry
Corresponding Author:

Tapan Das Bairagya,

F5 MO QTR, NBMC Campus,

Sushrutanagar Darjeeling-734012,

West Bengal, India.

E-mail: tdasbairagya@gmail.com

DOI: $10.14260 / \mathrm{jemds} / 2020 / 455$

How to Cite This Article:

Dhali S, Bairagya TD. A study on clinical profile and common comorbid conditions among chronic obstructive pulmonary disease (COPD) patients in a tertiary care hospital in Eastern India. J. Evolution Med. Dent. Sci. 2020;9(30):2085-2088, DOI: 10.14260/jemds/2020/455

Submission 28-01-2020,

Peer Review 20-06-2020,

Acceptance 26-06-2020,

Published 27-07-2020.

Copyright (C) 2020 JEMDS. This is an open access article distributed under Creative Commons Attribution License [Attribution 4.0 International (CC BY 4.0)] 


\section{BACKGROUND}

Chronic Obstructive Pulmonary Disease (COPD) is a major cause of chronic morbidity and mortality throughout the world, and is the fourth leading cause of death in the world. ${ }^{1}$ Chronic obstructive pulmonary disease (COPD) is a disorder that is characterized by progressive airflow limitation that is not fully reversible. In practice, this is measured as the $\mathrm{FEV}_{1} / \mathrm{FVC}$ ratio and the arbitrary definition of airflow obstruction is generally taken to be an $\mathrm{FEV}_{1} / \mathrm{FVC}$ ratio lower than $0.70 .^{2} \mathrm{~A}$ diagnosis COPD should be considered if any patient who has dyspnoea, chronic cough or sputum production and/or a history of exposure to risk factors for the disease, especially cigarette smoking. ${ }^{3}$ It is increasingly recognized that, many patients with COPD have co-morbidities that have a major impact on quality of life and survival. Airflow limitation and particularly hyperinflation affect cardiac function and gas exchange. Inflammatory mediators in the circulation may contribute to skeletal muscle wasting and cachexia and may initiate or worsen co-morbidities such as ischemic heart disease, heart failure, osteoporosis, normocytic anaemia, diabetes, metabolic syndrome, and depression. ${ }^{4}$ In our study we have tried to find out common co morbid condition like diabetes, hypertension, anaemia and their clinical profile. In our study we have tried to find out common co-morbid condition and presentation of COPD. It is very important to address the co-morbid condition when we are treating a COPD patient.

\section{METHODS}

This is prospective observational study conducted among One hundred (100) patients, clinicospirometrically diagnosed as COPD in West Bengal and its neighbourhood and also among referred patients from the nearby states from January 2010 to January 2011. All the patients attending a tertiary care Hospital in eastern India both inpatient and outpatient department and who have been diagnosed as COPD by spirometry with bronchodilator reversibility. Subjects were evaluated following a pre-designed case record format.

Initially 117 patients, who were attending to a tertiary care hospital in eastern India, diagnosed as COPD as per GOLD guideline. Among these 117 patients, 100 patients were taken after satisfying all the criteria for study. Then these 100 Patients were interrogated in detail about their disease history, examined clinically, and lastly detailed investigations were done for subsequent management. Informed consents were taken from all the patients, with approval from Institutional Ethics Committee.

\section{Inclusion Criteria}

- Those patients attending to chest OPD diagnosed to have COPD by pulmonary function test.

- Those patient diagnosed outside attending first time in our chest OPD.

- Those patients admitted in our inpatient department and diagnosed to have COPD.

\section{Exclusion Criteria}

- $\quad<40$ years of age.

- $\quad>12 \%$ post bronchodilator reversibility on spirometry.

- Family h/o asthma.

- Heart failure. (By Framingham criteria) S.

The study was undertaken in accordance with the following protocol-

1. A through history including socioeconomic status of the patient Presenting clinical feature.

2. Blood Pressure and fasting along with postprandial plasma glucose measure.

3. Presences of risk factors before admission were sought.

A detailed clinical examination including respiratory system was done. COPD patients commonly presented with-

- Dyspnoea that progressive, persistent, worse with exercise.

- Chronic cough, may be intermittent, may be unproductive.

- Chronic sputum production.

- H/o exposure to risk factors especially tobacco smoke, occupational dust or smoke from biomass fuel.
Investigations Done
a. Blood for $\mathrm{Hb} \%$, total and differential white cell count, platelets, ESR.
b. Fasting and post prandial blood sugar, serum urea, creatinine, liver function test, electrolytes.
c. Chest $\mathrm{x}$ ray PA view and appropriate lateral view when indicated.
d. Sputum examination for acid fast bacilli
e. ECG of all leads
f. PFT with bronchodilator reversibility in stable condition
g. Blood Pressure taken by sphygmomanometer.

\section{Study Tools}

Spirometer, Chest X-Ray (PA), Sphygmomanometer and Routine Blood Investigation

\section{Study Techniques}

One year observational study planned to assess the diagnosis and severity of COPD patients along with co morbidity as per GOLD-2009 guideline along with patient stratification as per socioeconomic pattern. Data obtained thereby will be analysed with the help of SPSS software version 16 to find out any correlation between severities of COPD with socioeconomic pattern of COPD patients. Appropriate statistical analysis will be done with the observed data.

\section{RESULTS}

In our study a total of 100 cases of COPD were taken who attended to the OPD and admitted in our hospital maintaining the inclusion and exclusion criteria and all the patients were diagnosed case of COPD according to GOLD guidelines. Among 
100 cases of COPD most of the cases were above 50 years age group (89\%) of which around $48 \%$ are above 65 years age group (Table-I). Among 100 COPD patients most of the patients were male (84\%) compare to female $(16 \%)$. The study shows most of the patients were above 50 years age group (As $89 \%$ male vs. $87.5 \%$ female) and among these most of the patients were above 65 years (48\% male vs. $50 \%$ female). The most common presenting complains were shortness of breath (SOB-100\%), cough (COU-86\%) then expectoration (EXP-54\%) (Diagram-II). In this study we found $10.7 \%$ of male COPD patient found to have anaemia in contrary to only $18.75 \%$ of female COPD patient having anaemia (TableII).

\begin{tabular}{|ccccc|}
\hline Age (Years) & Male & Percentage (\%) & Female & Percentage (\%) \\
$40-49$ & 9 & $11 \%$ & 2 & $12.5 \%$ \\
$50-64$ & 35 & $41 \%$ & 6 & $37.5 \%$ \\
$\geq 65$ & 40 & $48 \%$ & 8 & $50 \%$ \\
\hline \multicolumn{5}{c}{ Table I. Age and Sex Distribution of Patients } \\
\hline
\end{tabular}

\begin{tabular}{|ccc|}
\hline Residence & No. of Cases (Anaemia) & Percentage (\%) \\
Male (84 Cases) & 9 & $10.7 \%$ \\
Female (16 Cases) & 3 & $18.75 \%$ \\
\hline Table II. Anaemia in COPD Patients $(\mathbf{H b}<\mathbf{1 3} \mathbf{g} / \mathbf{d L}$ in Male and \\
Hb $<\mathbf{1 2} \mathbf{g} /$ dL in Female was taken as Cut-Off Value). \\
\hline
\end{tabular}

\begin{tabular}{|c|c|c|c|c|c|c|}
\hline \multirow{2}{*}{$\begin{array}{c}\text { Co morbidities } \\
\text { Sex }\end{array}$} & \multicolumn{3}{|c|}{$\begin{array}{c}\text { Diabetes } \\
\text { (FPG }>126 \mathrm{mg} / \mathrm{dL} / \\
\text { PPBG }>200 \mathrm{mg} / \mathrm{dL} \text { ) }\end{array}$} & \multicolumn{3}{|c|}{$\begin{array}{c}\text { Hypertension } \\
\text { (BP > 140/90 } \\
\text { mmHg) }\end{array}$} \\
\hline & Male & Female & Total & Male & Female & Total \\
\hline No. of Cases & 12 & 2 & 14 & 16 & 4 & 20 \\
\hline Percentage (\%) & $14 \%$ & $12.5 \%$ & $14 \%$ & $19 \%$ & $25 \%$ & $20 \%$ \\
\hline \multicolumn{7}{|c|}{ Table III. Incidence of Diabetes / Hypertension } \\
\hline
\end{tabular}

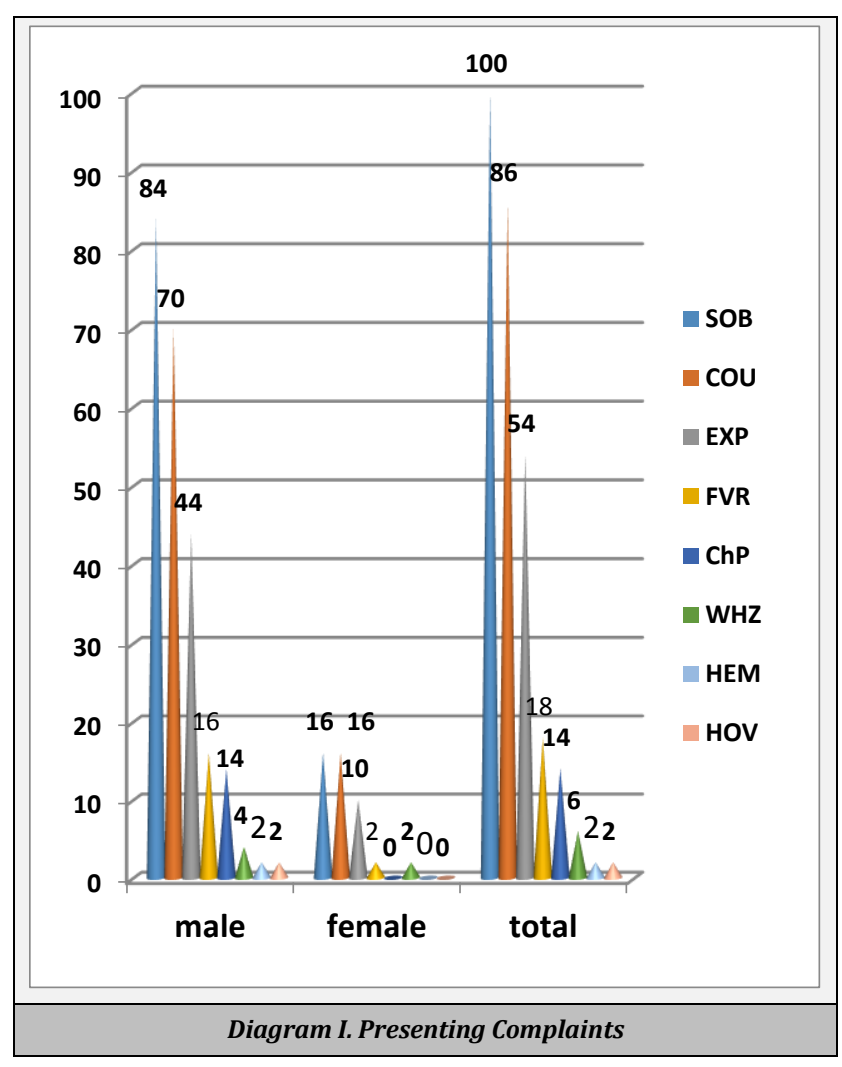

Anaemia of chronic disease and iron deficiency anaemia was commonly occurrence. In our study, it was found that, 14 cases of having diabetes (14\%) and 20 cases of having hypertension (20\%) but none of the patient in my study shows having diabetes and hypertension both. In this study there are 6 patient (7\%) male COPD patient having ischemic heart disease and $12.5 \%$ of female patient having ischemic heart disease by ECG criteria only. Regarding the severity of COPD patients are grouped according to GOLD guideline. In this study $40.5 \%$ of male patients belongs to severe COPD group. In this study there are 82 cases who are recent or past smoker. Out of these 82 cases, 80 cases are male and only 2 cases are female and among non-smoker 14 cases belong to female. Most of the patient presented with shortness of breath and cough with or without expectoration. Beside this few patients presented with fever, chest pain, wheezing. Only 2 patients have associated haemoptysis and 2 patients have hoarseness of voice (Diagram-I). Regarding nutritional status, we found most of the patients having average nutritional status (63\%). Among male patient $68 \%$ belong to average nutritional group and $22.5 \%$ poor nutritional group. But among female most of the female $(62.5 \%)$ belong to poor nutritional group. The severity of COPD patients is grouped according to GOLD guideline. In this study $40.5 \%$ of male patients belongs to severe COPD group compare to $50 \%$ female belong to severe COPD group. Regarding very severe COPD, $26 \%$ of the male patients are in this group compare to $12.5 \%$ of female.

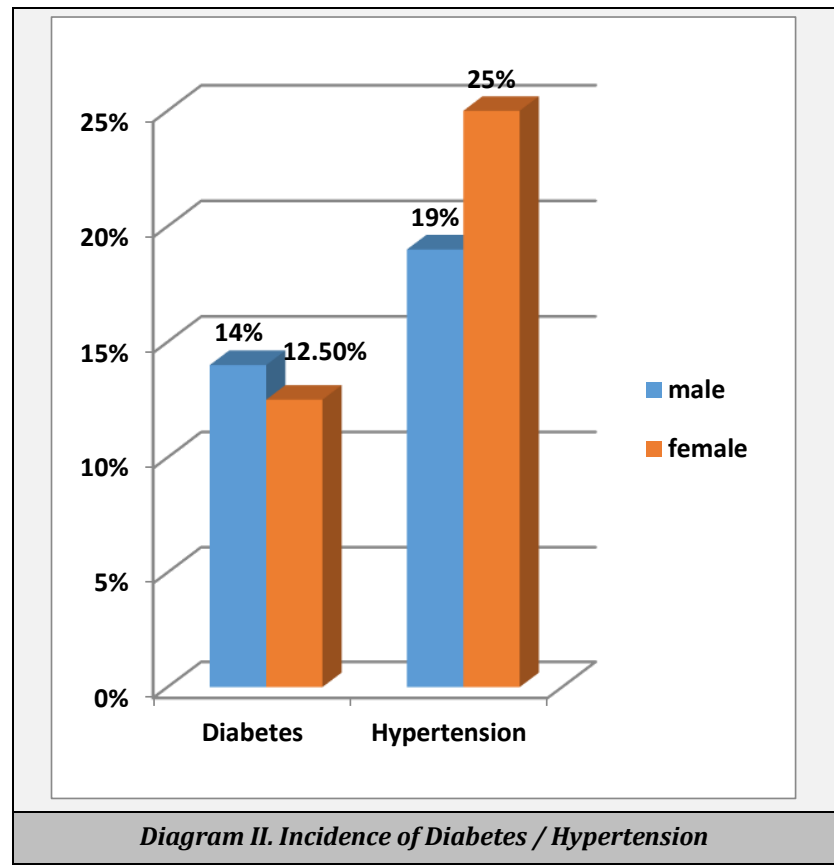

\section{DISCUSSION}

Epidemiological data suggest that certain co-morbid diseases including diabetes are much more common in patients with COPD and are associated with a worse COPD outcome. ${ }^{5}$ As shown by Cazzola et al., patients with COPD have a higher burden of Type II D. Mellitus. Sode et al showed that patients with COPD have a greater burden of not only DM but also of myocardial infarction, lung cancer, depression and hip fracture. In contrast to these findings, Korean researchers did not find any association between COPD and greater DM prevalence, which may be related to high percentage of underweight subjects in the studied population. ${ }^{6}$ In our study 
we have found that, 14 cases of having diabetes (14\%) and 20 cases of having hypertension (20\%) but none of the patient in my study shows having diabetes and hypertension both. In this hospital based study it was found a total of $14 \%$ cases of diabetes and $20 \%$ cases of hypertension. Among male the incidence of diabetes is $14 \%$ compare to $12.5 \%$ in female and regarding hypertension the incidence is in male is $19 \%$ compare to $25 \%$ in female. In our study we have tried to give a message to address the co-morbid condition and their occurrence in chronic obstructive pulmonary disease patients.

In the past studies by Díez Manglano J et al. ${ }^{7}$ showed in a Prospective and multicentre study that the most frequent co morbidities were heart failure (59\%), diabetes (48\%), myocardial infarction (29\%), moderate kidney failure (22\%), cerebrovascular disease (19\%), hypertension (71\%), anaemia $(62 \%)$, atrial fibrillation (34\%), dyslipidaemia (28\%) and obesity (21\%).

Another study conducted by D. M. Mannino et $\mathrm{al}^{8}$. in logistic regression models adjusting for age, sex, race, smoking, body mass index and education, subjects with GOLD stage 3 or 4 COPD had a higher prevalence of diabetes (Odds ratio (OR) 1.5, 95\% confidence interval (CI) 1.1-1.9), hypertension (OR 1.6, 95\% CI 1.3-1.9) and cardiovascular disease (OR 2.4, 95\% CI 1.9-3.0). In this study we found $10.7 \%$ of male COPD patient found to have anaemia in contrary to only $18.75 \%$ of female COPD patient having anaemia (Table-II). Anaemia of chronic disease and iron deficiency anaemia was commonly occurrence. In our study, it was found that, 14 cases of having diabetes (14\%) and 20 cases of having hypertension (20\%) but none of the patient in my study shows having diabetes and hypertension both. In this study there are 6 patient (7\%) male COPD patient having ischemic heart disease and $12.5 \%$ of female patient having ischemic heart disease by ECG criteria only

In our study among the total no of 100 patients, 84 cases (84\%) are male and 16 cases (16\%) are female. Regarding age distribution, most of the patients are above 50 years age group (as 89\% male vs. 87.5\% female) and among these most of the patients are above 65 years ( $48 \%$ male vs. $50 \%$ female).

In earlier studies by Kenneth R. Chapman ${ }^{9}$ et al. by a random sampling of 192 primary-care physicians (96 American and 96 Canadian; 154 men and 38 women) using a hypothetical case presentation and a structured interview. They showed that initially, COPD was given as the most probable diagnosis significantly more often for men than women ( $58 \%$ vs. $42 \%$; p $<0.05$ ). After spirometry, COPD diagnosis rates for men and women were $74 \%$ vs. $66 \%$ (p = not significant); after the steroid trial $85 \%$ vs. $79 \%$ ( $p=$ not significant).

A recent study Inga-Cecilie Sørheim ${ }^{10}$ et al. showed that female gender was associated with lung function reduction and more severe disease in subjects with COPD with early onset of disease or low smoking exposure. These findings may suggest a gender difference in susceptibility to the lungdamaging effects of cigarette smoking.

Another recent retrospective cohort study by Joan B Soriano ${ }^{11}$ et al. of British patients with COPD was constructed from the General Practice Research Database (GPRD) total of 50,714 incident COPD patients were studied, 23,277 (45.9\%) of whom were women. While prevalence rates of COPD in the UK seem to have peaked in men, they are continuing to rise in women. This trend, together with the ageing of the population and the long term cumulative effect of pack-years of smoking in women, is likely to increase the present burden of COPD in the UK. Bone mineral density was not measured in our study due to resource limitation.

\section{CONCLUSIONS}

Although COPD is primarily a disease of the respiratory system, systemic effects are varied and important components that can modify the prognosis of the disease and other health related aspects. In our study, we found that anaemia, diabetes, hypertension, ischemic heart disease, and malnutrition are the common comorbid conditions. Comorbid conditions are very common among COPD patients. Similarities of presentation often misguide us. We must search for co-morbid conditions among COPD patient.

Financial or Other Competing Interests: None.

\section{REFERENCES}

[1] Lozano R, Naghavi M, Foreman $K$, et al. Global and regional mortality from 235 causes of death for 20 age groups in 1990 and 2010: a systematic analysis for the Global Burden of Disease Study 2010. Lancet 2012;380 (9859):2095-128.

[2] Fishman AP, Elias JA, Fishman JA, et al. Fishman's pulmonary disease and disorders. Vol. I and Vol. II. $4^{\text {th }}$ edn New York: The MacGraw-Hill Companies 2008:729-46 (vol. I), 1517-34(vol. II).

[3] Rodriguez-Roisin R, Rabe KF, Anzueto A, et al. Pocket guide to COPD diagnosis, management and prevention: gold. 2008:7-8. http://www. goldcopd.

[4] Global strategy for diagnosis, management, and prevention of COPD. http//www.goldcopd.org

[5] Petty TL. The history of COPD. Int J Chron Obstruct Pulmon Dis 2006;1(1):3-14.

[6] Niranjan MR, Dadapeer K, Rashmi BK. Lipoprotein profile in patients with chronic obstructive pulmonary disease in a tertiary care hospital in South India. Journal of Clinical and Diagnostic Research 2011;5 (5):990-3.

[7] Díez-Manglano J, Bernabeu-Wittel M, Escalera-Zalvide A, et al. Comorbidity, discapacity and mortality in patients with multiple conditions and chronic obstructive pulmonary disease. Rev Clin Esp 2011;211 (10):504-10.

[8] Mannino DM, Thorn D, Swensen A, et al. Prevalence and outcomes of diabetes, hypertension and cardiovascular disease in COPD. Eur Respir J 2008;32 (4):962-9.

[9] Chapman KR, Tashkin DP, Pye DJ. Gender bias in the diagnosis of COPD. Chest 2001;119 (6):1691-5.

[10] Sørheim IC, Johannessen A, Gulsvik A, et al. Gender differences in COPD: are women more susceptible to smoking effects than men? Thorax 2010;65 (6):480-5.

[11] Soriano JB, Maier WC, Egger P, et al. Recent trends in physician diagnosed COPD in women and men in the UK. Thorax 2000;55 (9):789-94. 